\title{
A Bayesian Approach to Video Object Segmentation via Merging 3-D Watershed Volumes
}

\author{
Yu-Pao Tsai, Chih-Chuan Lai, Yi-Ping Hung, and Zen-Chung Shih
}

\begin{abstract}
In this letter, we propose a Bayesian approach to video object segmentation. Our method consists of two stages. In the first stage, we partition the video data into a set of three-dimensional (3-D) watershed volumes, where each watershed volume is a series of corresponding two-dimensional (2-D) image regions. These 2-D image regions are obtained by applying to each image frame the marker-controlled watershed segmentation, where the markers are extracted by first generating a set of initial markers via temporal tracking and then refining the markers with two shrinking schemes: the iterative adaptive erosion and the verification against a presimplified watershed segmentation. Next, in the second stage, we use a Markov random field to model the spatio-temporal relationship among the 3-D watershed volumes that are obtained from the first stage. Then, the desired video objects can be extracted by merging watershed volumes having similar motion characteristics within a Bayesian framework. A major advantage of this method is that it can take into account the global motion information contained in each watershed volume. Our experiments have shown that the proposed method has potential for extracting moving objects from a video sequence.
\end{abstract}

Index Terms-Markov random field, three-dimensional (3-D) watershed volume, video object segmentation, watershed segmentation.

\section{INTRODUCTION}

$\mathbf{V}$ IDEO object segmentation plays an important role in many advanced video applications (such as in MPEG-4 or in virtual reality), but still remains a challenging research topic.

A popular approach to video object segmentation is to combine a technique for single image segmentation with a temporal tracking procedure [20]. Unfortunately, single image segmentation is itself a very difficult problem (which may not be easier than video object segmentation). Other techniques in [12], [15] consider video sequences to be three-dimensional (3-D) signals and extend two-dimensional (2-D) methods to them, although the time axis does not play the same role as the spatial axis. The drawback of this technique is that a moving object in one frame must overlap with its corresponding object in the next frame. If the motion distance of the object is large, the object may become disconnected from one frame to the next. Most of the unsupervised segmentation algorithms only utilize low-level

Manuscript received October 8, 2002; revised April 21, 2004. This paper was recommended by Associate Editor M. Strintzis.

Y.-P. Tsai is with the Institute of Information Science, Academia Sinica, Taipei 115, Taiwan 30050, R.O.C., and is also with the Department of Computer and Information Science, National Chiao Tung University, Hsinchu 300, Taiwan 30050, R.O.C.

C.-C. Lai and Y.-P. Hung are with the Institute of Information Science, Academia Sinica, Taipei, Taiwan, R.O.C., and also with the Department of Computer Science and Information Engineering, National Taiwan University, Taipei 106, Taiwan 30050, R.O.C. (e-mail: hung@csie.ntu.edu.tw).

Z.-C. Shih is with the Department of Computer and Information Science, National Chiao-Tung University, Hsinchu, Taiwan 30050, R.O.C.

Digital Object Identifier 10.1109/TCSVT.2004.839973 features such as color, texture, motion, frame difference and histogram [9], [20]. However, without high-order information, semantic video object extraction is hard to achieve. Therefore, many researches have allowed a certain degree of human interaction. For example, the methods introduced in [2], [4] require some human interaction for the initial segmentation of the first image in the video. In fact, almost all the automatic algorithms developed for extracting video objects have some limitations. For example, the automatic method proposed in [20] can only extract homogeneous regions, instead of complete objects.

Realizing that there exists no generic automatic algorithm applicable to all kinds of video sequences, we focus on the problem of extracting video objects having similar motion characteristic. The method proposed in this letter consists of two stages: 1) generation of 3-D watershed volumes and 2) Bayesian merging of 3-D watershed volumes. Details of the two stages will bdescribed in Sections II and III. Experimental results will be shown in Section IV, and the conclusion will be given in Section V.

\section{GENERATION OF 3-D WATERSHED Volumes}

Watershed algorithm has been become popular technique for image segmentation [5], [13], [18]. In this letter, we apply watershed technique to video object segmentation by constructing 3-D watershed volumes. Given a video clip $\left\{I_{t}, 0 \leq t \leq T\right\}$ we can regard the data as one volume image. Our method first partitions the volume image into a set of 3-D watershed volumes, where each 3-D watershed volume is a series of corresponding 2-D image regions. Fig. 1 shows the flowchart of our method for generating 3-D watershed volumes. These 2-D image regions are obtained by applying to each image frame the marker-controlled watershed segmentation described in Step 2 of Section II-B. The procedure for generating 3-D watershed volumes can be divided into two phases: initial segmentation and temporal tracking. Details of these two phases are described below.

\section{A. Initial Segmentation}

In the initial phase, the first frame of the video clip $I_{0}$ is partitioned into a set of 2-D regions by applying the watershed segmentation algorithm to the gradient image of $I_{0}$. However, the basic watershed transformation tends to produce over-segmentation due to noise or local irregularities in the gradient image. Since overly segmented regions may not be reliable enough for the next phase of temporal tracking, we adopt a preprocessing method called "topographic simplification" to alleviate the over-segmentation problem. In our current implementation, the topographic surface of the gradient image is simplified by removing the local minima [19]. First, we apply a dilation operation with a structuring element of $2 \times$ 


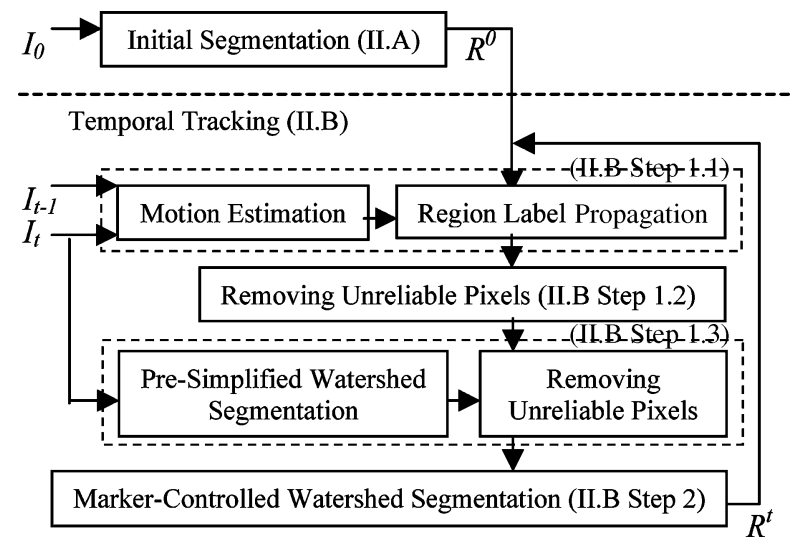

Fig. 1. Flowchart of generating 3-D watershed volumes.

2 pixels, i.e., let $g_{1}=\operatorname{Grad}\left(I_{0}\right) B_{2 \times 2}$. Next, we apply to $\operatorname{Grad}\left(I_{0}\right)$ a "reconstruction by erosion" [17] from $g_{1}+h$, i.e., let $g_{2}=\varphi^{(r e c)}\left[g_{1}+h, \operatorname{Grad}\left(I_{0}\right)\right]$. Notice that using a larger $h$ can eliminate more local minima. Finally, we can obtain a reasonable segmentation of $I_{0}$ by applying the basic watershed transformation to the simplified gradient image $g_{2}$.

In this letter, the above procedure of "topographic simplification followed by watershed transformation" will be referred to as the presimplified watershed segmentation, and will be applied again to each subsequent frame for the purpose of refining the extracted markers, as described in Step 1.3.

\section{B. Temporal Tracking}

In the second phase, our algorithm repeats the following two steps for each subsequent frame in the video clip: 1) marker extraction and 2) marker-controlled watershed segmentation. The task of marker extraction is to extract reliable seed regions based on the segmented regions obtained from the previous frame. Given these reliable markers, the marker-controlled watershed segmentation can not only accurately extract the boundaries of the watershed regions, but also can detect newly emerging regions.

Step1-Marker Extraction: Marker extraction is crucial to the success of the temporal tracking phase and deserves some special attention here. Our method for extracting markers consists of the following three substeps:

Step 1.1-Region label propagation by motion-based backward projection: First, initial markers are obtained by using backward pixel projection based on backward motion vectors. That is, for each pixel $\mathrm{p}$ in the current frame, we assign to the region label of the corresponding pixel in the previous frame to it. The correspondence is determined by using the backward motion vector $\mathbf{m}_{\mathbf{p}}$. Here, we choose to use backward motion to avoid generating empty and conflicting areas in the current frame. The dense field of backward motion vectors is estimated by using a template-matching algorithm that adopts adaptive windows, similar to the one used in [6]. To save the computation time, we first estimate a sparse field of motion vectors at every $4 \times 4$ pixel spacing. Then, the dense pixel-wise motion vectors are computed using bilinear interpolation. The approximation error can be dealt with the following process.

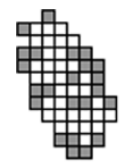

(a)

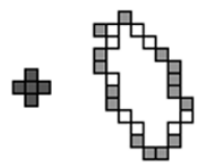

(b) (c)

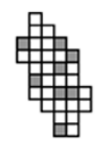

(d)

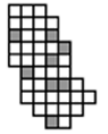

(e)

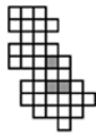

(f)
Fig. 2. Example of Step 1.2 for marker extraction. (a) Initial marker with unreliable pixels colored in grey. (b) Cross-shaped structuring element of 5 pixels. (c) Border pixels removed with the normal erosion. (d) Interior pixels obtained with the normal erosion. (e) Eroded marker after the first iteration of adaptive erosion. (f) After the second iteration (stable and stopped).

Step 1.2-Removing unreliable pixels from initial markers by iterative adaptive erosion: Since motion vectors are usually not very accurate, we must remove unreliable region assignments due to erroneous pixel correspondences. In order to reduce the possibility of generating false boundaries in the next substep, the extracted markers should be as large as possible, and completely contained in their true corresponding regions-which are unfortunately unknown to the computer.

Consider an initial marker $M_{i}$. A pixel $\mathrm{p} \in M_{i}$ is regarded as an unreliable pixel if it has an unreliable region propagation, that is, if $\bar{\varepsilon}(\mathbf{p})$ is greater than $k \cdot \bar{E}_{i}$, where $\bar{\varepsilon}(\mathbf{p})$ denotes the local mean of textural error centered round pixel $p$ (that is, the error of texture, including intensity and color, between the corresponding pixels)

$$
\bar{\varepsilon}(\mathbf{p})=\frac{1}{N_{U_{\mathbf{p}}}} \sum_{\mathbf{p}^{\prime} \in U_{\mathbf{p}}}\left|I_{t}\left(\mathbf{p}^{\prime}\right)-I_{t-1}\left(\mathbf{p}^{\prime}+\mathbf{m}_{\mathbf{p}^{\prime}}\right)\right|
$$

where $U_{\mathbf{p}}=\{\mathbf{p}$ and its eight neighbors having the same region label as $\mathrm{p}\}, N_{U_{\mathrm{p}}}$ is the number of elements in the set $U_{\mathbf{p}}$, and $\bar{E}_{i}$ denotes the global mean of textural error for the whole area of marker $M_{i}$

$\overline{E_{i}}=\min \left(\max \left(\frac{1}{N_{M_{i}}} \sum_{\mathbf{p} \in M_{i}}\left|I_{t}(\mathbf{p})-I_{t-1}\left(\mathbf{p}+\mathbf{m}_{\mathbf{p}}\right)\right|, 2\right), 16\right)$

where $N_{M_{i}}$ is the number of the pixels in marker $M_{i}$. The reason for constraining $\bar{E}_{i}$ to 2 and 16 is to prevent using an unreasonable large or unreasonable small threshold. The two numbers, 2 and 16 , are determined according to our experiments.

In this substep, we apply an iterative adaptive erosion to trim off "unreliable border pixels" of the initial markers, as illustrated in Fig. 2. The adaptive erosion (erode if "unreliable") is performed iteratively with a cross-shaped structuring element of 5 pixels, shown in Fig. 2(b), until the result becomes stable. Notice that the adaptively eroded marker shown in Fig. 2(e) is a union of the normally eroded marker [shown in Fig. 2(d)] and the reliable pixels, colored in white, are contained in the border portions [shown in Fig. 2(c)].

Note that using a lower $k$ can eliminate more marker pixels. In the case of foreground and background objects, which are not distinctive, $k$ should be set conservatively. We found that $k=1.2$ works well for most MPEG-4 test sequences in hand. The resulting markers with different values of $k$ using frame 116 of the "foreman" sequence are shown in Fig. 3. Pixels in black represent any undefined areas. 


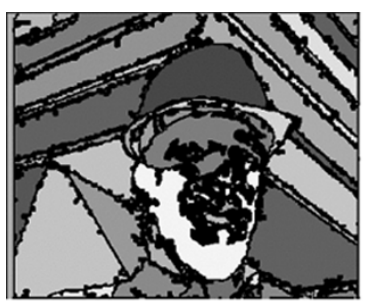

(a)

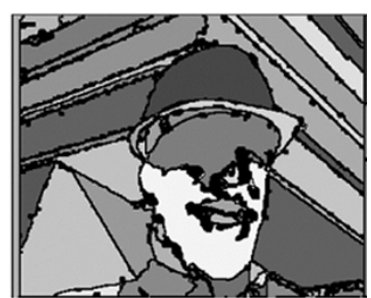

(b)
Fig. 3. Markers extracted from frame 116 of sequence "foreman" with different the value of $k$ after Step 1.2 for marker extraction. (a) $k=0.8$. (b) $k=1.2$.

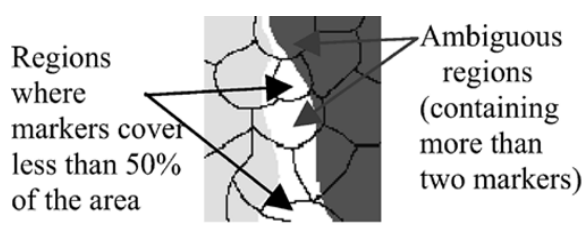

(a)

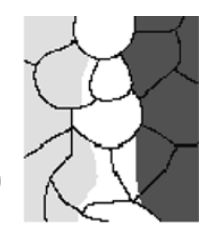

(b)
Fig. 4. Example of Step 1.3 for marker extraction. (a) Two different markers are overlaid by the watershed lines obtained from presimplified watershed segmentation. (b) The shrunk marker after removing the doubtful portions.

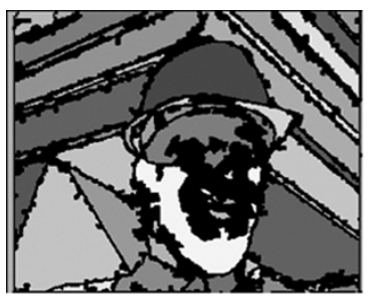

(a)

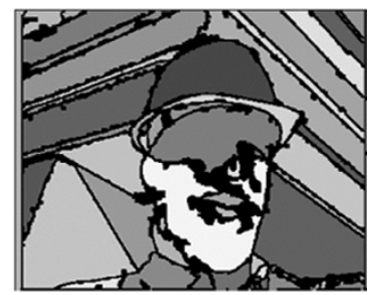

(b)
Fig. 5. Markers extracted from frame 116 of sequence "foreman" with different the value of $k$ after Step 1.3 for marker extraction. (a) $k=0.8$. (b) $k=1.2$

Step 1.3-Removing unreliable pixels by checking with a presimplified watershed segmentation: Here, we first generated a reasonably fine segmentation of the current frame by applying the presimplified watershed segmentation described in Section II-A, with a small value of parameter $h$. For each generated watershed region, check if it contains only one marker and the sole marker occupies more than half of the watershed region. If so, the sole major marker will be retained for driving the marker-controlled watershed segmentation in the next step. Otherwise, the marker pixel in this watershed region will be considered "unreliable," and will be removed from the markers, as illustrated in Fig. 4. Fig. 5 shows the final markers obtained by applying this substep to the markers shown on Fig. 3. We can see that after this step, small and ambiguous pieces of the marker are removed.

Step 2-Marker-Controlled Watershed Segmentation: Based on the reliable markers obtained from the last step, we can then extract more precise region boundaries by using the markercontrolled watershed segmentation [8], [20]. One problem accompanying marker-controlled segmentation is that no newly exposed regions can be extracted without creating new markers. To solve this problem, we modify the marker-controlled watershed algorithm slightly. For the flooding process of the markercontrolled watershed algorithm used in [20], when the water

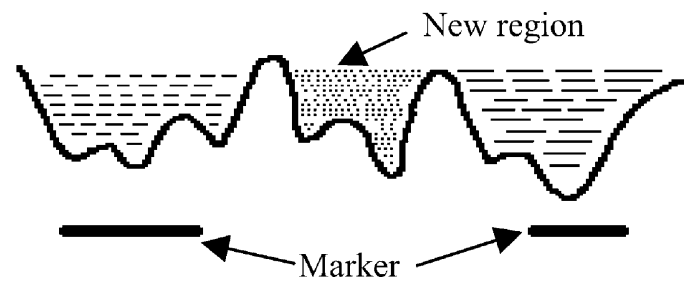

Fig. 6. New region is labeled if the dynamics of a catchment basin exceeds a certain threshold.

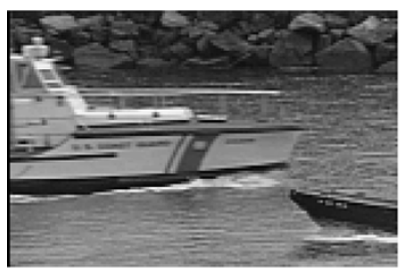

(a)

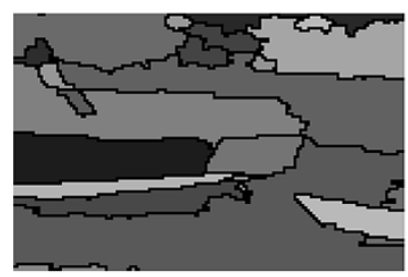

(c)

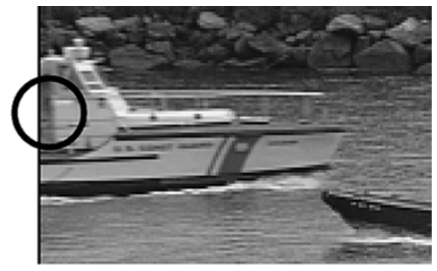

(b)

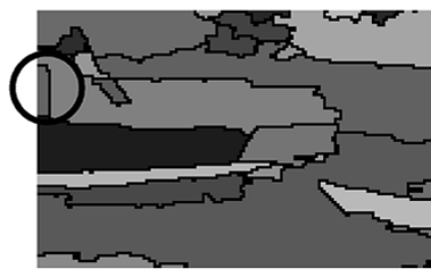

(d)
Fig. 7. Demonstration of detecting new region by using dynamic thresholding. (a) Frame 26. (b) Frame 27. (c) Segmented result of frame 26. (d) Segmented result of frame 27

coming from two different basins is about to meet, the two basins are merged if "both have the same label" or "at least one of them is unlabeled." Our modification for creating new markers is if the dynamics of an unlabeled basin larger than a certain threshold [7], [10], the basin will be given a new label (Fig. 6). Fig. 7 shows the result of detecting new regions using frame 26 and 27 of the "coastguard" sequence. The big boat is entering the image from the left, and the background water can be detected as a new region.

\section{BAYESIAN MERGING OF WATERSHED VOLUMES}

Once the 3-D watershed volumes are generated, as described in Section II, we need to merge them into a set of desired video objects. Here, we propose a Bayesian approach to merging watershed volumes having similar motion characteristics, hoping that more global motion information can be utilized within a formal framework. Here, we use a Markov random field (MRF) to model the spatial and temporal relationships among different watershed volumes. A closely related work is the one done by Gelgon and Bouthemy, which uses region-level MRFs to track a spatial image partition [3]. Another work proposed by Patras et al. [12] labels watershed segments by MAP. The labeling criterion is the maximization of the conditional a posteriori probability of the labeling field given the motion hypothesis, the estimate of the label field of the previous frame, and image intensities. However, our method is different from theirs, not only in how the MRF is applied (we employ the MRF after tracking while they do it before tracking), but also in how the class-conditional probability is modeled. 


\section{A. Extraction of Features From 3-D Watershed Volume}

Before applying the Bayesian merging to 3-D watershed volumes, the representative features for each watershed volume need to be extracted. Motion information is an important cue to produce semantic objects. Hence, for each watershed volume $v$, we construct a feature vector $\boldsymbol{\theta}_{v}$ based on motion information. We first decompose each watershed volume $v$ into a set of regions $\left\{R_{v}^{t} \mid 0 \leq t_{b}(v) \leq t \leq t_{e}(v) \leq T\right\}$, where $R_{v}^{t}$ denotes a region which can be obtained by intersecting frame $t$ with the watershed volume $v, t_{b}(v)$ and $t_{e}(v)$ are the indices of the beginning frame and the ending frame of the watershed volume $v$, respectively. Note that the indices of the beginning and ending frames of the watershed volumes can vary for the watershed volume $v$ due to the appearance or disappearance of objects in the scene.

In practical situations, image motion of a rigid object can be approximately modeled by a small number of motion parameters. If two regions roughly correspond to the same 3-D rigid object, the motion parameter should be about the same. From the above observation, we compute a motion parameter vector $\boldsymbol{\theta}_{v}^{t}$ for each region $R_{v}^{t}$ by applying the least-median squares (LMedS) robust estimator [13] to the backward dense motion field obtained from Step 1.1 of Section II-B. The motion parameters can be estimated by

$$
\hat{\boldsymbol{\theta}}_{v}^{t}=\arg \min _{\boldsymbol{\theta}_{v}^{t}}\left\{\operatorname{median}_{\mathbf{p} \in R_{v}^{t}}\left\|\mathbf{m}_{\mathbf{p}}^{t}-\mathbf{u}\left(\mathbf{p} ; \boldsymbol{\theta}_{v}^{t}\right)\right\|\right\}
$$

where $\mathbf{u}($.$) is a parameterized motion field, \|\cdot\|$ is defined as the two-norm operator, and $\mathbf{m}_{\mathbf{p}}^{t}$ is the motion vector of pixel $p$ in frame $t$. After the parameters for all the regions in the watershed volume $v$ are determined, we can construct a motion feature vector: $\boldsymbol{\theta}_{v}=\left[\boldsymbol{\theta}_{v}^{t_{b}(v)}, \boldsymbol{\theta}_{v}^{t_{b}(v)+1}, \ldots, \boldsymbol{\theta}_{v}^{t_{e}(v)}\right]$. Notice that the dimensionality of $\boldsymbol{\theta}_{v}$ is $\left(t_{e}(v)-t_{b}(v)+1\right) \cdot d$, where $d$ is the dimension of $\boldsymbol{\theta}^{t}$. In our current implementation, the motion characteristics of $R_{v}^{t}$ are described by a constant motion field, that is, $\mathbf{u}\left(\mathbf{p} ; \boldsymbol{\theta}_{v}^{t}\right)=\boldsymbol{\theta}_{v}^{t}$, where $\boldsymbol{\theta}_{v}^{t}=\left[m_{x}^{t}, m_{y}^{t}\right]$ and $m_{x}^{t}$ and $m_{y}^{t}$ are the coordinates of the mean motion vector.

\section{B. Proposed Method}

In this work, we assume that the number of video objects $N$ to be extracted (including the background objects) is known. Given a set of 3-D watershed volumes $V=\left\{v_{j}, j=1, \ldots, K\right\}$, where $K$ is the number of 3-D watershed volumes, a volume adjacency graph (VAG) can be constructed to express the neighborhood relationship among 3-D watershed volumes. Each node in the graph corresponds to a watershed volume, and between two volumes exists an arc if the volumes are spatially connected. Next, we define a label field $L=\left\{l_{v} \mid l_{v} \in[1 \ldots N], v \in \mathrm{V}\right\}$ on the VAG. Given $M=\left\{\boldsymbol{\theta}_{v} \mid v \in V\right\}$, we estimate the labeling field $L$ by maximizing the a posteriori probability (MAP). Using the Bayes rule, the a posteriori probability density function can be expressed as

$$
P(L \mid M) \propto P(M \mid L) \cdot P(L) .
$$

The first term on the right-hand side of (4) is the conditional probability distribution $P(M \mid L)$. It is modeled as a Gaussian distribution, which implies that each object should have minimum motion variance

$$
P(M \mid L) \propto \exp \left(-\sum_{v \in V} \frac{1}{2 \sigma_{l}^{2}} \sum_{t=t_{b}(v)}^{t_{e}(v)}\left\|\boldsymbol{\theta}_{v}^{t}-\boldsymbol{\mu}^{t}\left(l_{v}\right)\right\|^{2}\right)
$$

where $\boldsymbol{\mu}^{t}\left(l_{v}\right)$ is the mean of the parameter vectors of all watershed volumes in frame $t$ whose corresponding labels are $l_{v}, \sigma_{l}$ is a function of the size of the video object.

The second term on the right-hand side of (4) is the prior probability distribution $P(L)$, which is a regularization term. To take into account the "degree" of adjacency between two watershed volumes, we directly extend a measure of adjacency degree between two regions proposed in [3] to that between two watershed volumes

$$
b\left(v_{j}, v_{k}\right)=\frac{\ell_{v_{j}, v_{k}}}{\ell_{v_{j}, v_{k}}+\left\|g_{j}-g_{k}\right\|}
$$

where $\ell_{v_{j}, v_{k}}$ is the area of the common border between $v_{i}$ and $v_{j}$, and $g_{j}$ and $g_{k}$ are the gravity centers of $v_{j}$ and $v_{k}$, respectively. We model the prior as a Gibbs distribution. Before defining a Gibbs distribution, we need to define the cliques. Here, only two-site cliques are considered and straightforwardly obtained from the arc of the VAG. Let $\boldsymbol{C}_{v}$ be the set of all binary cliques. The Gibbs distribution is given by

$$
P(L)=\frac{1}{Z_{b}} \exp \left(-U_{b}(L)\right)
$$

where $Z_{b}$ is a normalizing constant and $U_{b}(L)$, the regularization potential, is defined as

$$
U_{b}(L)=\sum_{\left(v_{j}, v_{k}\right) \in C_{v}}-b\left(v_{j}, v_{k}\right) \cdot \delta\left(l_{v_{j}}, l_{v_{k}}\right)
$$

where $\delta($.$) is a Kronecker delta function. The regularization$ term tends to favor identical labels for two neighboring volume sites.

The maximum a posteriori probability (MAP) estimate of $L$ is obtained by minimizing the following energy function

$$
\begin{gathered}
\hat{L}=\arg \min _{L}\left(\sum_{v \in V} \frac{1}{2 \sigma^{2}} \sum_{t=t_{b}(v)}^{t_{e}(v)}\left\|\boldsymbol{\theta}_{v}^{t}-\boldsymbol{\mu}^{t}\left(l_{v}\right)\right\|^{2}\right. \\
\left.+\sum_{\left(v_{j}, v_{k}\right) \in C_{v}}-b\left(v_{j}, v_{k}\right) \cdot \delta\left(l_{v_{j}}, l_{v_{k}}\right)\right) .
\end{gathered}
$$

Energy minimization is performed using an ICM algorithm proposed by Besag [1], sometimes also called the greedy algorithm. At each iteration, each volume sites is visited. The label of each site is either changed to the label that yields maximal decrease of the energy function, or left unchanged if no energy reduction is possible. The process stops when no more changes can be made. The detail can be found in [16].

\section{EXPERIMENTAL RESULTS}

In this section, we use the "foreman", and "coastguard" sequences, shown in Figs. 8 and 9, respectively, to demonstrate the performance of our algorithm. The experiments are run on 


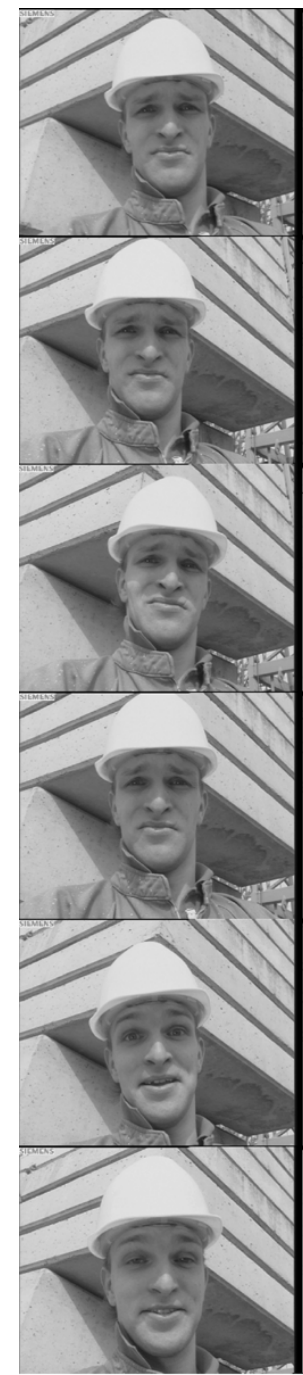

(a)

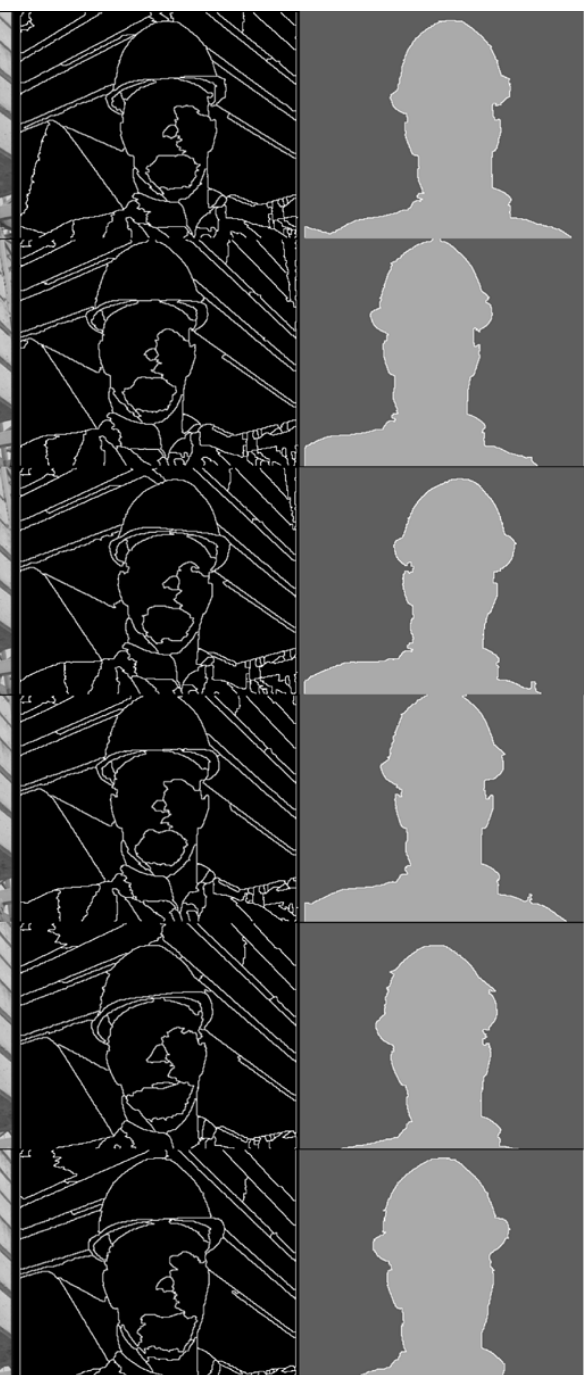

(b)

(c)
Fig. 8. "Foreman" sequence: frame 1, 20, 40, 60, 80, 100. (a) Original images. (b) After temporal tracking. (c) After Bayesian merging.

AMD Athlon 1.2 GHz PC with $384 \mathrm{MB}$ RAM. The sizes of the "foreman" sequence and the "coastguard" sequence are 352 $\times 288$ and $352 \times 240$. As to the total execution time, the processing of the "foreman" sequence with 100 frames requires 483 $\mathrm{s}$ and the "coastguard" sequence with 50 frames requires 131 s. In our current implementation, the gradient images are computed on a weighted YUV color space, i.e., $w_{y} Y+w_{u} U+w_{v} V$. The weighting factors, $w_{y}, w_{u}$, and $w_{v}$, are set to one, two, and two, respectively, to emphasize the color components.

In the "foreman" sequence, the human body has a moderate motion and the camera is moving as well. It can be seen from Fig. 8(b), where cross-sections of watershed volumes are shown, that the results obtained by marker-controlled temporal tracking look pretty good. By setting $N=2$ (i.e., the number of video objects to be extracted is 2 ), the watershed volumes depicted in Fig. 8(b) can be correctly merged into two video objects: the foreman and the background, as shown in Fig. 8(c). In this sequence, we have found that the similarity between the motions of the head and the shoulder could be more easily detected when

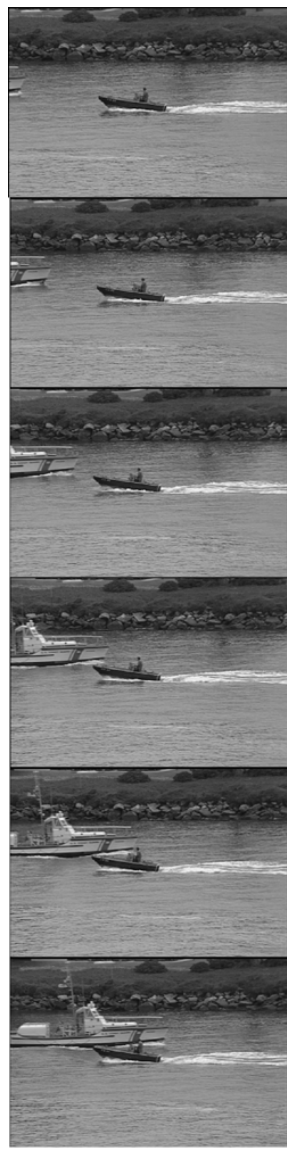

(a)

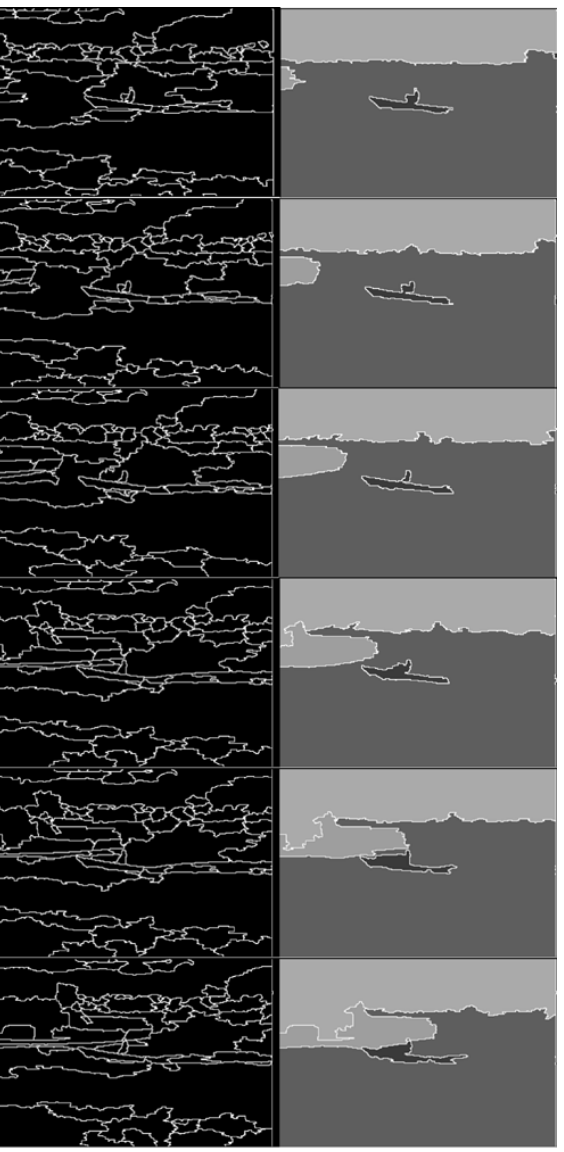

(b) (c)
Fig. 9. "Coastguard" sequence: frame 1, 10, 20, 30, 40, 50. (a) Original Images. (b) After temporal tracking. (c) After Bayesian merging.

considering a longer sequence. Therefore, our method can obtain better segmentation results than those obtained by Moschni et al. [9].

In the "coastguard" sequence, the horizontal camera drift is present while two boats are moving with different velocities and directions. Notice that the bigger boat is entering the image from the left, and its new emerging regions can be successfully extracted, as shown in Fig. 9(b). If we set $N=4$, the proposed Bayesian method can partition the video clip into four different objects: the bigger boat, the smaller boat, the water and the shore, as shown in Fig. 9(c). Compared with the results using the method proposed by Patras et al. [12], the segmented boundaries we extracted are much closer to the objects.

\section{CONCLUSION}

In this letter, we have proposed a new method for video object segmentation. This method first partitions the video data into a set of 3-D watershed volumes, and then extracts video objects by merging motion-coherent watershed volumes within a Bayesian framework. One major contribution of this work is that it models the prior information with a MRF over a volume adjacency graph (VAG), where each node of the VAG is a 3-D watershed volume and, hence, is able to take into account the global motion information contained in each watershed volume. 
This method is appropriate for extracting objects having similar motion because it can merge 3-D watershed volumes having similar motion with a Bayesian framework. Another contribution is that this letter proposes an efficient way to extract reliable markers by shrinking with two schemes: the iterative adaptive erosion and the verification against a presimplified watershed segmentation. Experimental results have shown that the proposed method has potential for extracting moving objects from a video sequence.

\section{REFERENCES}

[1] J. Besag, "On the statistical analysis of dirty pictures," J. R. Stat. Soc. B, vol. 48, no. 3, pp. 259-302, 1986.

[2] D. Gatica-Perez, G. Gu, and M.-T. Sun, "Semantic video object extraction using four-band watershed and partition lattice operators," IEEE Trans. Circuits Syst. Video Technol., vol. 11, no. 5, pp. 603-618, May 2001.

[3] M. Gelgon and P. Bouthemy, "A region-level motion-based graph representation and labeling for tracking a spatial image partition," Pattern Recognit., vol. 30, no. 4, pp. 725-740, 2000.

[4] C. Gu and M. Lee, "Semiautomatic segmentation and tracking of semantic video objects," IEEE Trans. Circuits Syst. Video Technol., vol. 8 , no. 5, pp. 572-584, May 1998.

[5] K. Haris, S. N. Efstratiadis, N. Maglaveras, and A. K. Katsaggelos, "Hybrid image segmentation using watersheds and fast region merging," IEEE Trans. Image Process., vol. 7, no. 12, pp. 1684-1699, Dec. 1998.

[6] T. Kanade and M. Okutomi, "A stereo matching algorithm with an adaptive window: theory and experiment," IEEE Trans. Pattern Anal. Mach. Intell., vol. 16, no. 9, pp. 920-932, Sep. 1994.

[7] C. Lemarechal and C. Fjortoft, "Comments on "Geodesic saliency of watershed contours and hierarchical segmentation'," IEEE Trans. Pattern Anal. Mach. Intell., vol. 20, no. 7, pp. 762-763, Jul. 1998.
[8] F. Meyer and S. Beucher, "Morphological segmentation," J. Vis. Commun. Image Representat., vol. 1, pp. 21-46, 1990.

[9] F. Moscheni, S. Bhattacharjee, and M. Kunt, "Spatiotemporal segmentation based on region merging," IEEE Trans. Pattern Anal. Mach. Intell., vol. 20, no. 8, pp. 897-915, 1998.

[10] L. Najman and M. Schmitt, "Geodesic saliencey of watershed contours and hierarchical segmenation," IEEE Trans. Pattern Anal. Mach. Intell., vol. 18, no. 12, pp. 1163-1173, Dec. 1996.

[11] M. Pardas and P. Salembier, "3-D morphological segmentation and motion estimation for image sequences," Signal Process., vol. 38, pp. 31-43, 1994.

[12] I. Patras, E. A. Hendriks, and R. L. Lagendijk, "Video segmentation by MAP labeling of watershed segments," IEEE Trans. Pattern Anal. Mach. Intell., vol. 23, no. 3, pp. 326-332, Mar. 2001.

[13] J. B. T. M. Roerdink and A. Meijster, "The watershed transform: definitions, algorithms and parallelization strategies," Fund. Inform., vol. 41, pp. 187-228, 2000.

[14] P. J. Rousseeuw, "Least median of squares regression," J. Amer. Statist. Assoc., vol. 79, pp. 871-880, 1984.

[15] P. Salembier and M. Pardas, "Hierarchical morphological segmentation for image sequence coding," IEEE Trans. Image Process., vol. 3, no. 5, pp. 639-651, Sep. 1994.

[16] Y.-P. Tsai, C.-C. Lai, Y.-P. Hung, and Z.-C. Shih, "A Bayesian Approach to Video Object Segmentation via Merging 3-D Watershed Volumes," Academia Sinica, Taiwan, R.O.C., Tech. Rep. TR-IIS-04-005, 2004.

[17] L. Vincent, "Morphological grayscale reconstruction in image analysis: application and efficient algorithms," IEEE Trans. Image Process., vol. 2, no. 2, pp. 176-201, Feb. 1993.

[18] L. Vincent and P. Soille, "Watersheds in digital spaces: an efficient algorithm based on immersion simulations," IEEE. Trans. Pattern Anal. Mach. Intell., vol. 13, no. 6, pp. 583-598, Jun. 1991.

[19] D. Wang, "A multiscale gradient algorithm for image segmentation using watersheds," Pattern Recognit., vol. 30, no. 12, pp. 2043-2052, 1997.

[20] — "Unsupervised video segmentation based on watersheds and temporal tracking," IEEE Trans. Circuits Syst. Video Technol., vol. 8, no. 5, pp. 539-546, May 1998. 